Supplementary materials for

\title{
Lead-Free Bilayer Thick Films with Giant Electrocaloric Effect near Room Temperature
}

Jinglei Li ${ }^{\dagger}, \#$, Yunfei Chang*, \#, Shuai Yang ${ }^{\dagger}$, Ye Tian ${ }^{\dagger}$, Qingyuan $\mathrm{Hu}^{\dagger}$, Yongyong Zhuang ${ }^{\dagger}$, Zhuo $\mathrm{Xu}^{\dagger}, \mathrm{Fei} \mathrm{Li}^{*}, \dagger$

$\dagger$ Key Laboratory of the Ministry of Education and International Center for Dielectric Research, School of Electronic Science and Engineering, Xi'an Jiaotong University, Xi'an 710049, China

\# Condensed Matter Science and Technology Institute, School of Instrumentation Science and Engineering, Harbin Institute of Technology, Harbin 150080, China.

*Corresponding author: changyunfei@hit.edu.cn (Y. C.); ful5@xjtu.edu.cn (F. L.) 


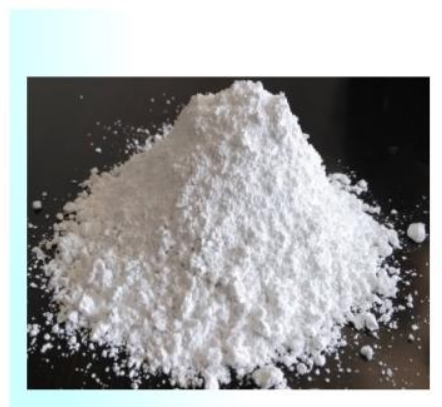

Calcined Powders

\section{Casted tape}

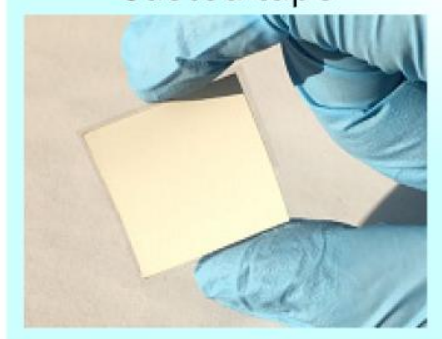

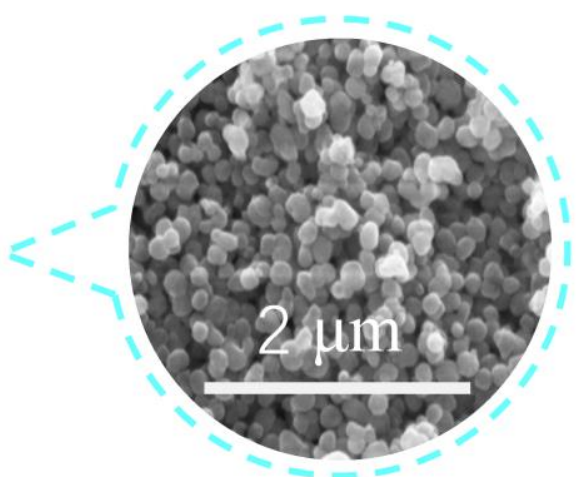

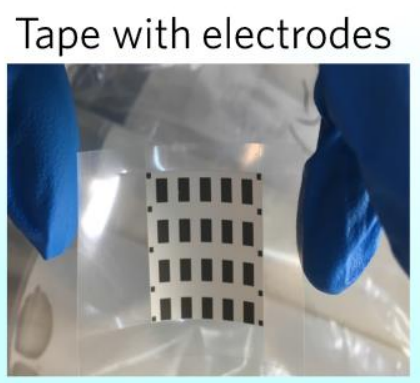

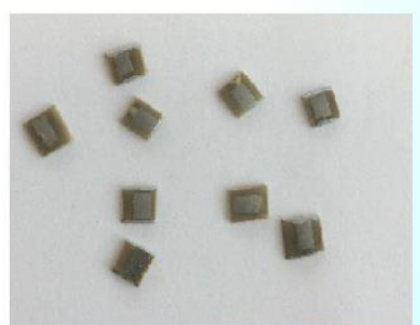

Sintered samples

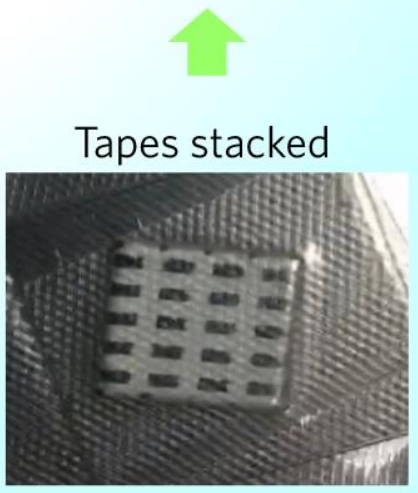

Figure S1. Calcined powder with the SEM image, green tape prepared by tape casting, tape with printed platinum electrodes, stacked green samples, and co-fired bilayer thick films were selected to illustrate the fabrication process of BZT-BST bilayer thick films. 


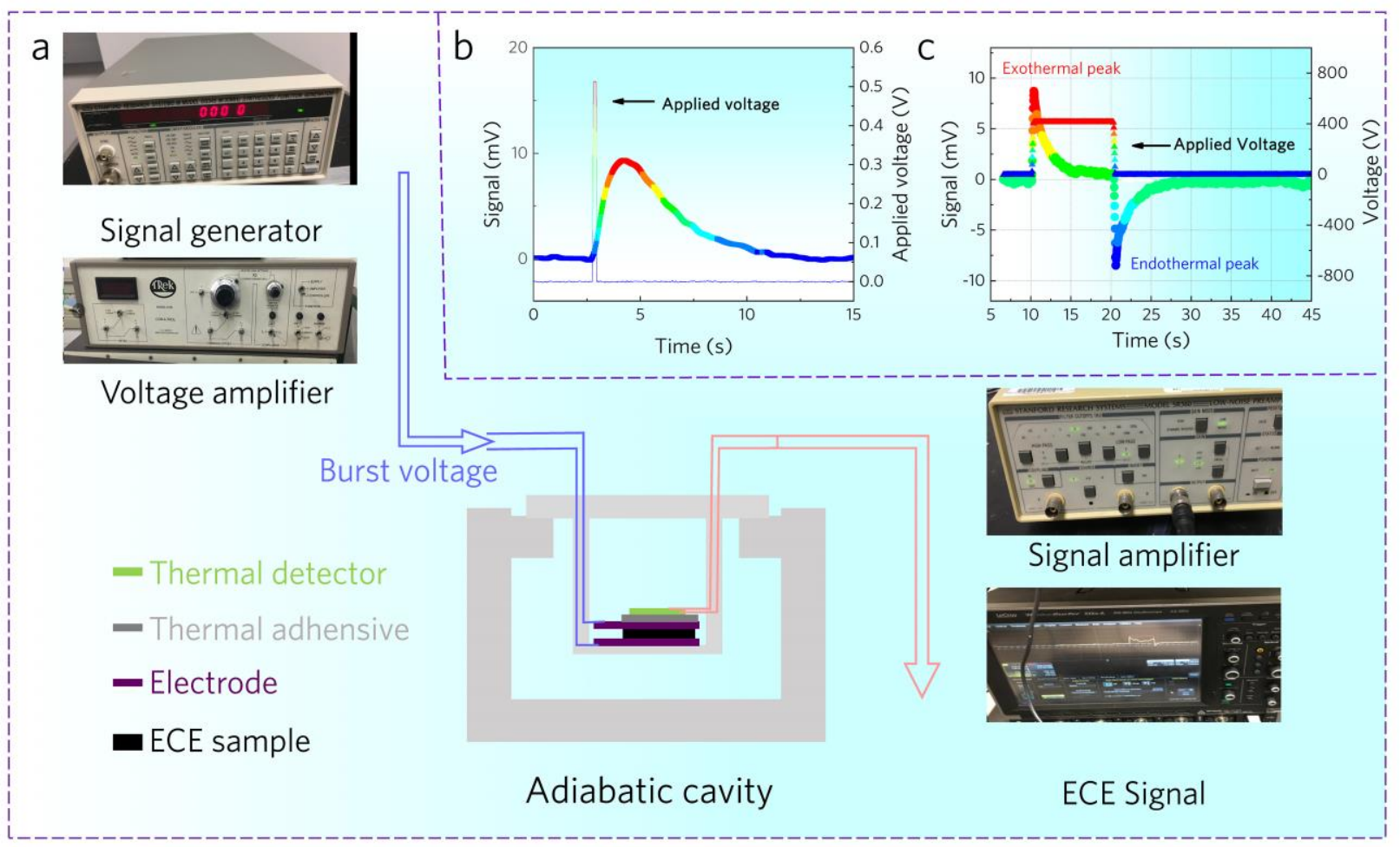

Figure S2. (a) Illustration of ECE direct measurement setup. (b) A typical recorded heat flux sensor signal representing $Q_{\mathrm{r}}$ generated from the reference resistor $R$ under a square voltage pulse. (c) A typical recorded heat flux sensor signal representing $Q_{\mathrm{ECE}}$ generated/absorbed by the bilayer BZTBST thick film under an applied square voltage. 